\title{
An Improved Inter-frame Difference Algorithm and its Application on Tidal Bore Video Auto Detection
}

\author{
Rui-Rong Wang ${ }^{1}$, Xu Zeng ${ }^{1}$, Cui-Fei Xu' ${ }^{1}$, Guo-Wen Zhang ${ }^{1}$, Ying-Hong Sun ${ }^{2}$, Yi Liu ${ }^{2}$ \\ ${ }^{1}$ Hangzhou Dianzi University, Hangzhou, Zhejiang Province, China \\ ${ }^{2}$ Hangzhou Hydrology and Water Resources Monitoring Station, Hangzhou, Zhejiang Province, China \\ wangrr@hdu.edu.cn
}

\begin{abstract}
As the mother river of Zhejing Province in the east of China, Qiantang River catch dozens of people's lives every year because of the strong tidal wave. In order to prevent the tidal disaster of Qiantang River, a real-time tidal bore detection and warning system must be built up. In this paper, an improved inter-frame difference algorithm based on Sobel image edge detection technique was presented, based on this this algorithm an intelligent video surveillance system was designed also. Experiments showed that the improved inter-frame algorithm was highly efficient in tidal bore detection not only in detection result but also the detection speed and efficiency. The system included a couple of video servers run on $\mathrm{Ti}$ DM6446 DSP and one client run on Windows PC. With this detection system, we have implemented Tidal bore detection and warning system in Qiantang River Successfully.

Index Terms - DM6446, intelligent video surveillance system, tidal detection, improved inter-frame difference algorithm
\end{abstract}

\section{Introduction}

Being such an important river as West Lake to Hangzhou city, Qiantang River run through the middle of the city, and its impact on people's daily life and their environment is deep and significant. However, there also exist some potential safety problems that may easily result in accidents. As the major natural disaster in Zhejiang Province (east China), "tidal disaster" has been proposed as the main technical issue during the eleventh five-year plan of China. Therefore, it's a great challenge for us to prevent the tidal disaster and make it serve for the development of the country.

Presently, many scholars domestic and abroad have carried out extensive research on tidal bore and made some achievements. Lin et al studied the formation, evolution and dissipation of the tidal bore, as well as the formation mechanism and model of the tidal bore, which analyze the dynamic change of water level and tidal current velocity in different condition [1]. Wolanskia et al studied the hydrodynamics of the undular tidal bore in the Daly Estuary of Northern Australia according to the field observations [2]. Huang et al studied the hydrodynamics of tidal bore, and carried out a physical modeling study in a rectangular flume with considerations of the tidal bore heights, the propagation speeds, the tidal current velocities, the front steepness, and the bore shapes, which simulate the formation of tidal bore in different initial conditions[3]. Pan et al successfully set up a 2D mathematical model with the Kinetic Flux Vector Splitting (KFVS) scheme after their analysis of the characteristics of the tidal bore in the Qiantang River Estuary with the field data [4].

Although there is a great deal of theoretical researches about tidal bore, it can play only a small role in preventing tidal disaster. With the rapid development of technology, we can make full use of modern communications networks, automatic test systems, embedded devices and computer vision technology to build a modern tidal detection and automatic warning system, with 24-hours automatic detection and realtime warning, which we have devoted a lot of effort to. As to automatic tidal bore detection, we have developed a system based on audio frequency recognition as well as a single-shaft tidal current velocity measuring device and a double-shaft tidal current velocity measuring device, all getting corresponding nation patents [5-7]. To realize real-time tidal bore warning of Qiantang River, we put forth a new method of predicting tidal water level and time based on neural network, as well as a method of real-time tidal bore prediction by dividing the river into many segments $[8,9]$.

However, when put into application, the previous methods proved to be unpractical. For instance, as Qiantang River is both high in sediment concentration and salinity, the contact device such as water-level meter is vulnerable to erosion and requires periodic maintenance. Meanwhile, as the tidal bore has such a great power, they are easily to be damaged. So it is urgent for us to develop a new automatic tidal bore detecting system which is non-contact. In this paper, we introduce an intelligent video surveillance system which applies image processing technology and video surveillance technology to achieve real-time monitoring of Qiantang River's tidal bore.

Over the years, video surveillance system has gone through from analog to digital, and becomes networked and intelligent. It is widely believed that video surveillance technology has undergone three stages [10], the third of which is intelligent digital video surveillance system based on embedded technology. With its high quality and efficiency, it is widely used in medical apparatus and instruments, robot vision system, intelligent transportation, safety protection and so on. For instance, optical tracking and infrared tracking technology has been applied to military field for missile guidance, gun control and range measurement [11]. Petrovskaya and Thrun have proposed an automatic vehicle detecting and tracking system for autonomous urban driving, enabling the real-time intelligent analysis of urban transports system [12]. Remagnino et al. have proposed a multi-agent surveillance system for use in monitoring scenes involving animate (pedestrians) and inanimate (vehicles) objects [13]. Hsieh et al presents a novel posture classification system that analyzes human movements directly from video sequences, making human movement analysis a very efficient and accurate 
process [14].

\section{Introduction of Video-Processing Algorithm}

The video-processing algorithm is critical for Intelligent Video Surveillance System. And video processing includes video coding and detecting the front end of the tidal bore through video analyzing. In this section, we first introduce different Video Coding methods and choose H.264 as the most suitable for our system. Then we present background subtraction, the basic inter-frame difference algorithm and the improved inter-frame difference algorithm. At last, we compare the three algorithms, and come to a conclusion that the improved inter-frame difference algorithm is the best one in tidal bore detecting.

\section{A. Video Coding}

Video Coding technique derives from the improvement of the performance of video capture devices. As video capture devices collect video with better quality and higher image definition, the data volume of video information expands, making the bandwidth condition and storage media of transport network even more demanding. To facilitate data storage and transmission, it is necessary to encode and compress the huge amounts of data. Then researchers take up research on video data, attempting to find out relevant information between consecutive points of both intra-frame and inter-frame, thus getting rid of redundant information in the video, which is the prototype of intra-frame prediction and inter-frame. According to the difference between compression algorithm, International Telecommunication Union, International Standard Organization and International Electro technical Commission begin to establish standards of compressed encoding audio and video [15-18].

Video encoding experts affiliated to International Telecommunication Union proposed standards such as H.261, H.263, and H.263++ successively. Moving Picture Experts Group which is affiliated to International Standard Organization and International Electro technical Commission proposed multimedia encoding standards such as MPEG-1, MPEG-2, and MPEG-4. The fundamentals of video encoding is removing video redundancy, reducing the size of video data by predictive encoding, transform encoding and statistical encoding, so as to realize data compression and reducing network data transfer [19-21].

The main characteristic of H.263 is its ability to provide favorable image quality when network load is heavy, and it is highly fault-tolerant and adaptive. H.263+ and H.263++ are the enhanced version of H.263. They are downward compatible to H.263 and meanwhile improve the encoding efficiency and error-resistance performance. However, the changes are small as they just improve under the framework of H.263 [22].

H.264 is a new digital video encoding standard developed by Joint Working Group on Video. The Group consists of Video Coding Experts Group from International Telecommunication Union and Moving Pictures Experts Group from International Standard Organization and International Electro technical Commission. The two expert groups jointly develop a video coding technology with high performance [23]. Like former standards, H.264 is a Hybrid coding mode of Differential Pulse Coding and Transform Coding. However, the coding system is divided into Video Coding Layer (VCL) and Network Abstraction Layer (NAL).VCL consists of VCL encoder and VCL decoder, accomplishing compressed encoding and decoding respectively. And VCL includes motion compensation unit, predictive encoding unit, transform encoding unit and entropy encoding unit and so on. NAL defines the packet-based data interface between video codec and external network, so as to integrate the video streaming of VCL to transport layer after protocol encapsulation [24]. H.264 adopts a concise design and strengthens the adaptive capacity to various kinds of channels. Its extensible network structure and grammar make it easy to deal with code error and packet loss in specific environment. Meanwhile, the application range is relatively wide, satisfying requirement of different image resolutions, different frame rates and different transmission and storage situations [25].

\section{B. Background Subtraction}

Background subtraction is a widely used method to extract a relatively intact target image. It is obvious that the detection effect mainly depend on background modeling method and background model updating technique. So it is a major subject in the field of Intelligent Video Surveillance System to build the background model quickly and accurately [26].

To build a proper background model, background subtraction generally adopt mixture Gaussian model which build multiple normal distributions for each pixel to describe the background [27]. Mixture Gaussian modeling algorithm can remove the noise that is caused by the long-time repeating movement of background, but it is easy to produce optical interference noise and can not eliminate the smearing created by tidal bore movement in a short time. So it produces no good recognition result for fast moving objects.

\section{Basic Inter-frame Difference Algorithm}

Unlike background subtraction, inter-frame difference algorithm can detect moving objects effectively. It detects and withdraws the target mainly by recognizing the difference between two successive frames of the video sequences and carrying out differential operation on video sequences [28].

The basic inter-frame difference algorithm can detect and extract moving targets using the difference of two or more consecutive frame in video sequence. It is based on the idea that pixels in the moving target area will have significant change between former and later frame. So we compare the frames to mark the pixels which have changed significantly. The regions of these pixels are considered as moving targets.

Assume $f_{i}$ and $f_{i+1}$ are the two adjacent images of $i$ and $i+1$ in the video sequence. They are defined as:

$$
\begin{gathered}
f_{i}=b_{i}(x, y)+m(x, y)+n_{i}(x, y) \\
f_{i+1}=b_{i+1}(x, y)+m(x+\Delta x, y+\Delta y) \\
+n_{i+1}(x, y)
\end{gathered}
$$


where $b_{i}(x, y), b_{i+1}(x, y)$ are the background of the image of $i$ and $i+1$ respectively, $m(x, y)$ and $m(x+\Delta x, y+\Delta y)$ are the moving target of the two images, $\Delta x$ and $\Delta y$ are motion vectors, while $n_{i}(x, y)$ and $n_{i+1}(x, y)$ are the noise of two images. And $g(x, y)$ which is the result of differencing the two images, is given by:

$$
\begin{aligned}
g(x, y)= & {[m(x+\Delta x, y+\Delta y)-m(x, y)] } \\
& +\left[b_{i+1}(x, y)-b_{i}(x, y)\right] \\
& +\left[n_{i+1}(x, y)-n_{i}(x, y)\right]
\end{aligned}
$$

From the above equation, $g(x, y)$ contains some noise $\left[n_{i+1}(x, y)-n_{i}(x, y)\right]$ and moving part $[m(x+\Delta x, y+\Delta y)$ $-m(x, y)]+\left[b_{i+1}(x, y)-b_{i}(x, y)\right]$. The difference image is considered as moving target if it is greater than the movement threshold prospect $T$.

The basic inter-frame difference algorithm can detect moving targets in ideal condition. However, it is especially susceptible to optical interference noise because of its sensitivity to the variation of light. So in practical application, it is rarely used as an isolated algorithm for the detecting of moving targets.

\section{Improved inter-frame difference algorithm}

In consideration of the limitation of the basic inter-frame difference algorithm, it is necessary to improve it for a better detecting performance. An improved inter-frame difference algorithm which combines image edge detection algorithm and the basic inter-frame difference algorithm is proposed here to overcome optical interference noise which is common in tidal detection circumstance. It is derived from the characteristic that Sobel edge detection algorithm is less affected by illumination changes. The core idea is to extract edge image of background frame and current frame through Sobel edge algorithm and take the difference to get the difference image of edge detection. Then we combine it with the inter-frame differencing result image by using the operation of "and" to get the moving targets. Fig .1 shows the process of the improved inter-frame difference algorithm.

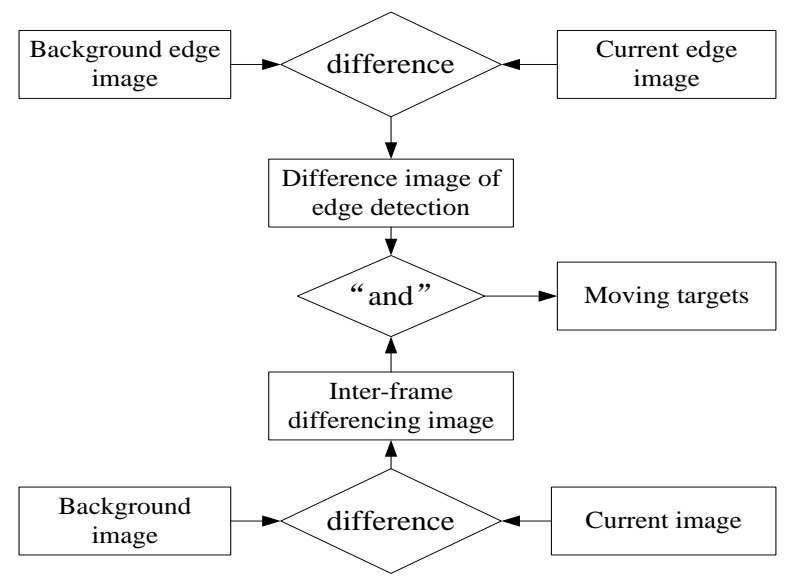

Fig. 1 The Process of the Improved Inter-frame Difference Algorithm
Assume $P(x, y)$ is the current edge image and $B(x, y)$ is the background edge image, while the value 1 stands for the point being an edge point while value 0 stands for not being. The difference operator of edge detection is defined as:

$$
D(x, y)=P(x, y)-B(x, y)
$$

We can analyze the result value of $D(x, y)$ as follows:

(1) if $D(x, y)$ is equal to 1 , the point of current frame is a moving target edge point;

(2) if $D(x, y)$ is equal to -1 , it is a non-moving target edge point;

(3) if $D(x, y)$ is equal to 0 , it must be the case of $P(x, y)=B(x, y)=0$ or $P(x, y)=B(x, y)=1$. In the former case, it isn't a moving target edge point. In the latter, we have two cases. One is that the point is the background edge both in the background frame and the current frame, so it is not moving target edge. The other is that the point is background edge both in background frame and the moving target edge in current frame, that is to say, the edge of moving target is overlapped with the background edge. So it is considered as non-moving target edge point.

From the analysis, we can draw the conclusion that it is only when $D(x, y)$ is equal to 1 that the point of current frame is a moving target edge point. So the difference image of edge detection is given by:

$$
d(x, y)= \begin{cases}255, & D(x, y)=1 \\ 0, & \text { other }\end{cases}
$$

Moving target image can be obtained by the operation of "and" between difference image of edge detection and interframe difference image.

Sobel algorithm is a mature edge detection algorithm which is simple and can produce good detection results. It provides smoothing effect of the noise and can provide accurate edge information to restore the contours of objects. The principle of Sobel is to use two operators (the vertical direction operator and the horizontal direction operator) for convolution operation. Convolution weights in both directions are shown in Table I. And the gradient value of each pixel is gotten by seeking the root-mean-square of the value in the corresponding position of two matrices. At last, compare the gradient value to the threshold $\mathrm{T}$. The point is identified as object edge points if it is greater than $\mathrm{T}$, and vice versa.

As Sobel edge detection algorithm is not sensitive to the variation of light, improved inter-frame difference algorithm can not only detect quickly, but also overcome optical interference noise effectively. Fig. 2 and Fig. 3 show the results of Sobel edge detection before and after the light intensity changes respectively.

TABLE I Sobel Operator

\begin{tabular}{|l|l|l|}
\hline-1 & 0 & 1 \\
\hline-2 & 0 & 2 \\
\hline-1 & 0 & 1 \\
\hline
\end{tabular}

\begin{tabular}{|c|c|c|}
\hline-1 & -2 & -1 \\
\hline 0 & 0 & 0 \\
\hline 1 & 2 & 1 \\
\hline
\end{tabular}




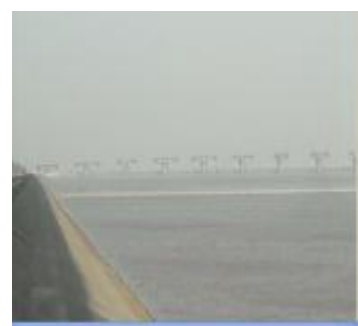

(a) Original Image

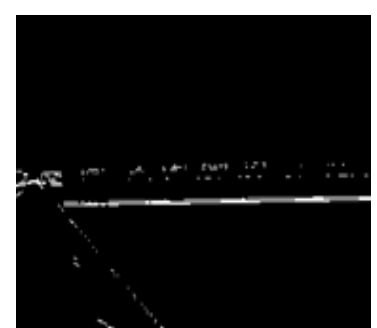

(b) Sobel Edge Detection Image

Fig. 2 Sobel Detection Result before Light Intensity Changes

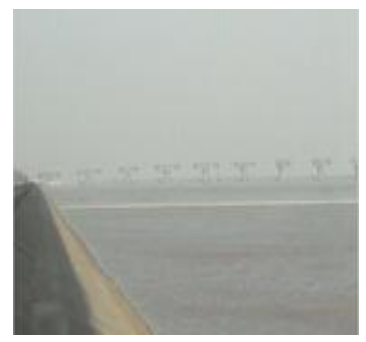

(a) Original Image

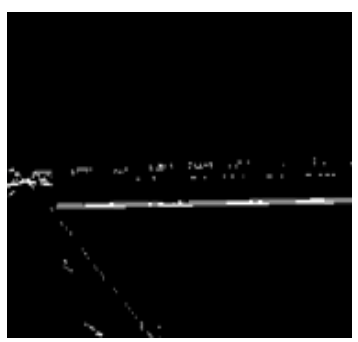

(b) Sobel Edge Detection Image

Fig. 3 Sobel Detection Result after Light Intensity Changes

The two figures illustrate that using Sobel edge detection, the image changes little in the changing of light intensity. In other words, the light intensity change has no effect on Sobel edge detection. Therefore, it can be concluded that Sobel edge detection algorithm performs better inhibitory effect of illumination change.

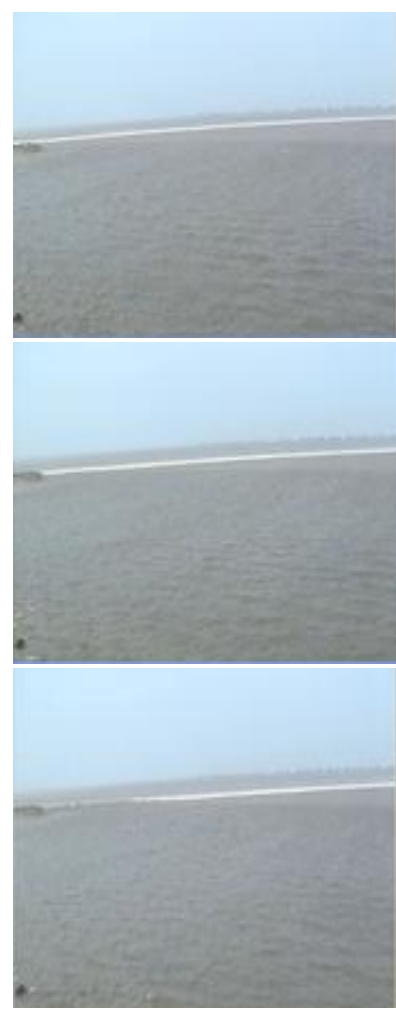

(a) Original Image

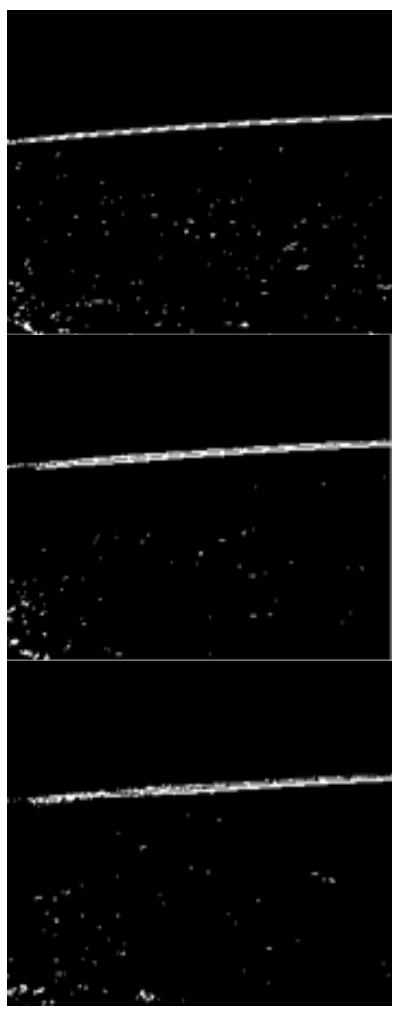

(b) Basic Inter-frame Difference

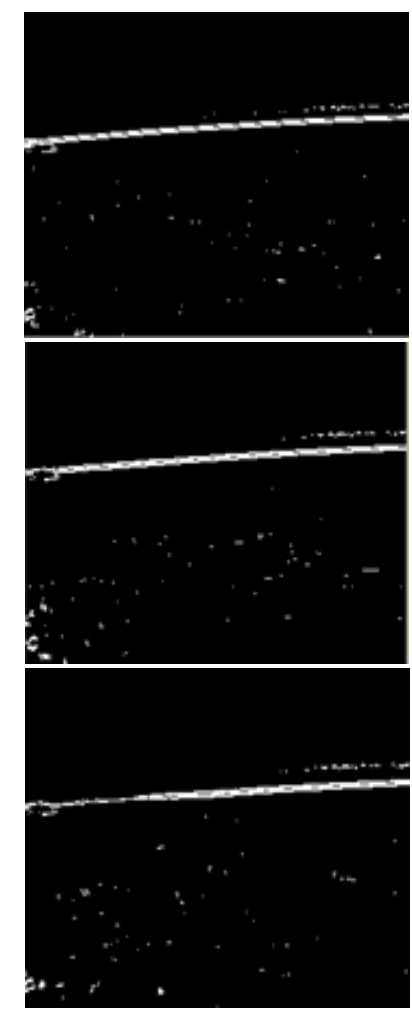

(c) Gaussian Mixture modeling

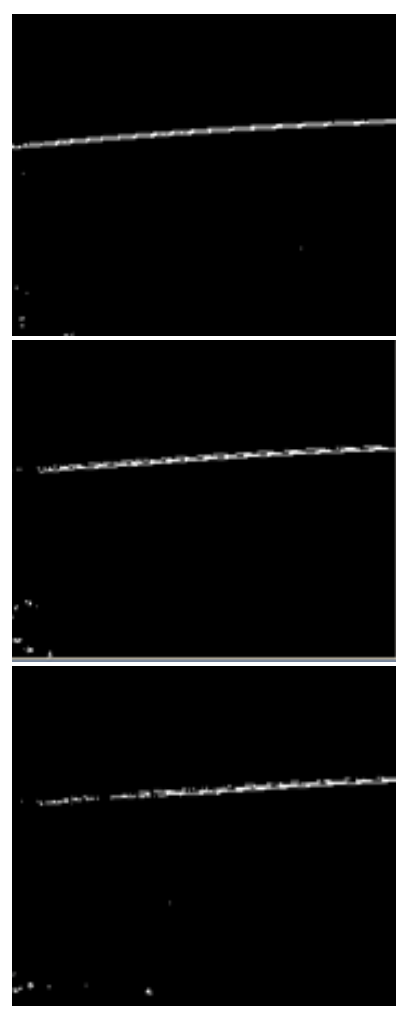

(d) Improved Inter-frame difference

Fig. 4 The Tidal Bore Recognition Results of Jiubao. 


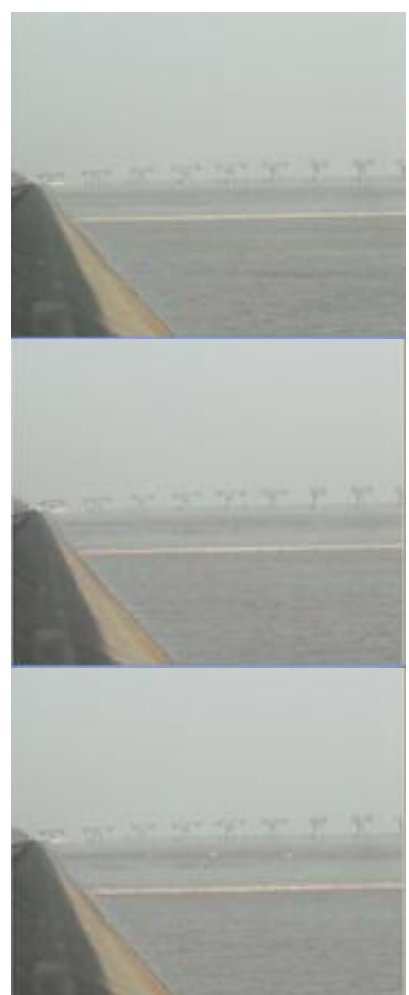

(a) Original Image

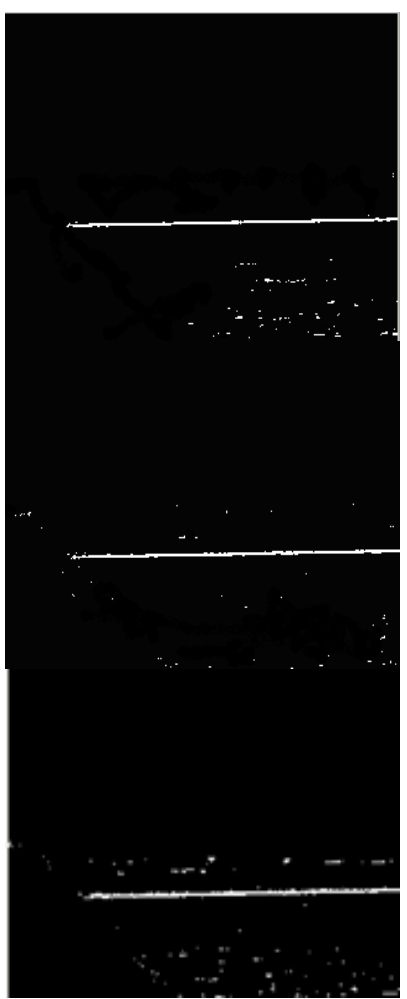

(b) Basic Inter-frame Difference

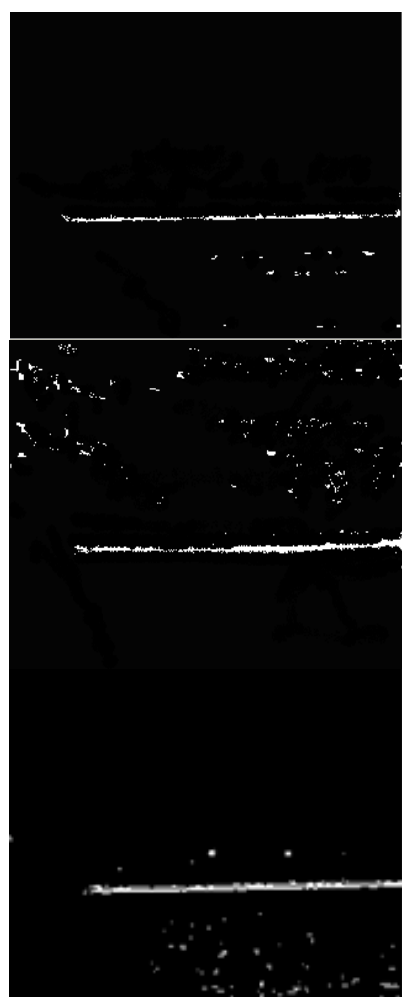

(c) Gaussian Mixture Modeling

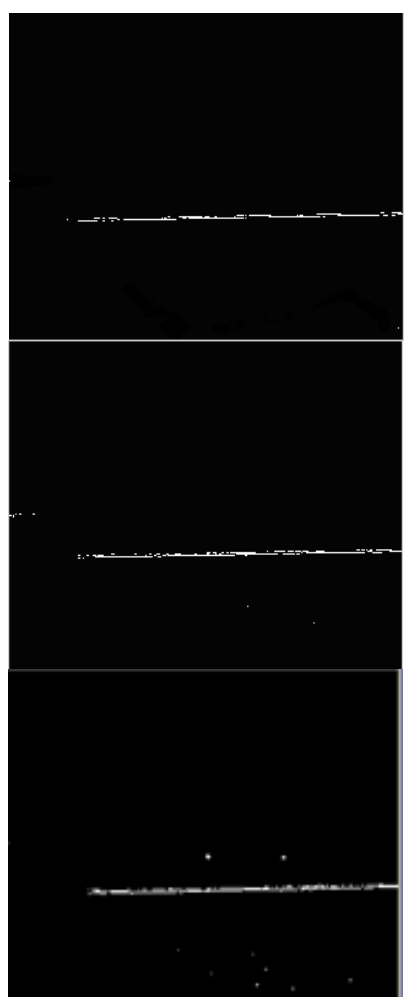

(d) Improved Inter-frame Difference

Fig. 5 The Tidal Bore Recognition Results of Jiangdong Bridge

TABLE II Algorithm Running Time (s)

\begin{tabular}{|c|c|c|c|c|}
\hline Algorithm & Original time & $\begin{array}{c}\text { Basic inter- } \\
\text { frame difference }\end{array}$ & $\begin{array}{c}\text { Gaussian mixture } \\
\text { modeling }\end{array}$ & $\begin{array}{c}\text { Improved inter- } \\
\text { frame difference }\end{array}$ \\
\hline Tidal video of Qibao & 75 & 31.65 & 88.35 & 33.75 \\
\hline $\begin{array}{c}\text { Tidal video of Jiangdong } \\
\text { Bridge }\end{array}$ & 47 & 18.95 & 55.15 & 20.15 \\
\hline Tidal video of Jiubao & 39 & 16.73 & 49.55 & 17.65 \\
\hline Average time & 53.67 & 22.44 & 64.35 & 23.85 \\
\hline
\end{tabular}

In order to further validate the performance of algorithms, an experiment is conducted to test the operating speed of those three algorithms in the same environment and with the same device configuration. The experiment result is shown in Table II .

Table II shows the basic inter-frame difference algorithm runs the fastest while the improved inter-frame difference algorithm runs at almost the same average speed of the basic inter-frame difference algorithm. As can be seen from the data in Table II, the improved inter-frame difference algorithm runs twice or triples faster than Gaussian mixture modeling.

It is concluded that Gaussian mixture modeling algorithm and the basic inter-frame difference algorithm are more easily influenced by weather, light, and other environment factors. So it is impractical for them to detect moving tidal bore in such complex environment as Qiantang River. The improved inter-frame difference algorithm, however, can effectively detect the arrival of the Qiantang
River's tidal bore with almost the same run time. Meanwhile, the low complexity can insure that the system can work stably on low-power embedded platform and basically meet the requirements of real-time monitoring.

\section{System Implementation}

The system is based on C/S (client / server) model, with the video server running on DM6446 and the client on PC. Fig. 6 shows the architecture of the intelligent video surveillance system. In this section, we first introduce DM6446 as it plays a key role in the system. Then we present our design of video server and client separately.

\section{A. Video processing subsystem}

As an intelligent video surveillance system developed for the tidal detection of Qiantang River, it not only inherits the inherent role and function of traditional video surveillance system, but also joins a more effective moving target detection algorithm to improve the performance and enhance security. 
As to execute the algorithm precisely, the requirement of the hardware is quite demanding.

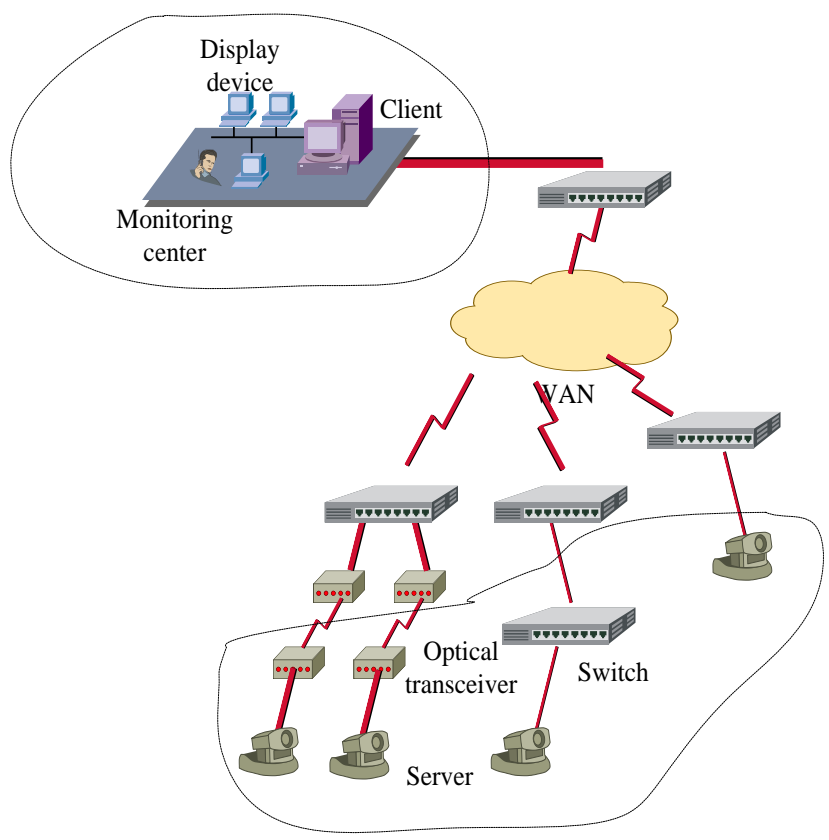

Fig. 6 System Architecture
Fig. 7 shows the hardware structure of DM6446, which is the video processing subsystem. As the video server of system, DM6446 consists of many different modules. The video capture module is to acquire the original video in UYVY format for surveillance and analysis. The video analysis module is to receive video signal from the video capture module, and detect the moving target in a designated area. If a moving target appears, a red circle will be drawn around it to remind the monitoring personnel of the abnormal situation. To reduce the data volume, a video coding module is used to encode the data passed from the analysis module with the coding algorithm called H.264 which is embedded in the DSP. After completing the encoding, the video coding module will store the encoding result on the hard driver and inform the transmission module whether the data is available. The function of the video transmission module include sending encoded video to the client over the network and receiving control commands from the client in order that the monitoring personnel can achieve control over the video server.

\section{B. The implementation of video server}

The video server conduct a sequence of operations, including video capture, video analysis, video encode, video store and video transmission. Fig. 8 shows the architecture of the video server.

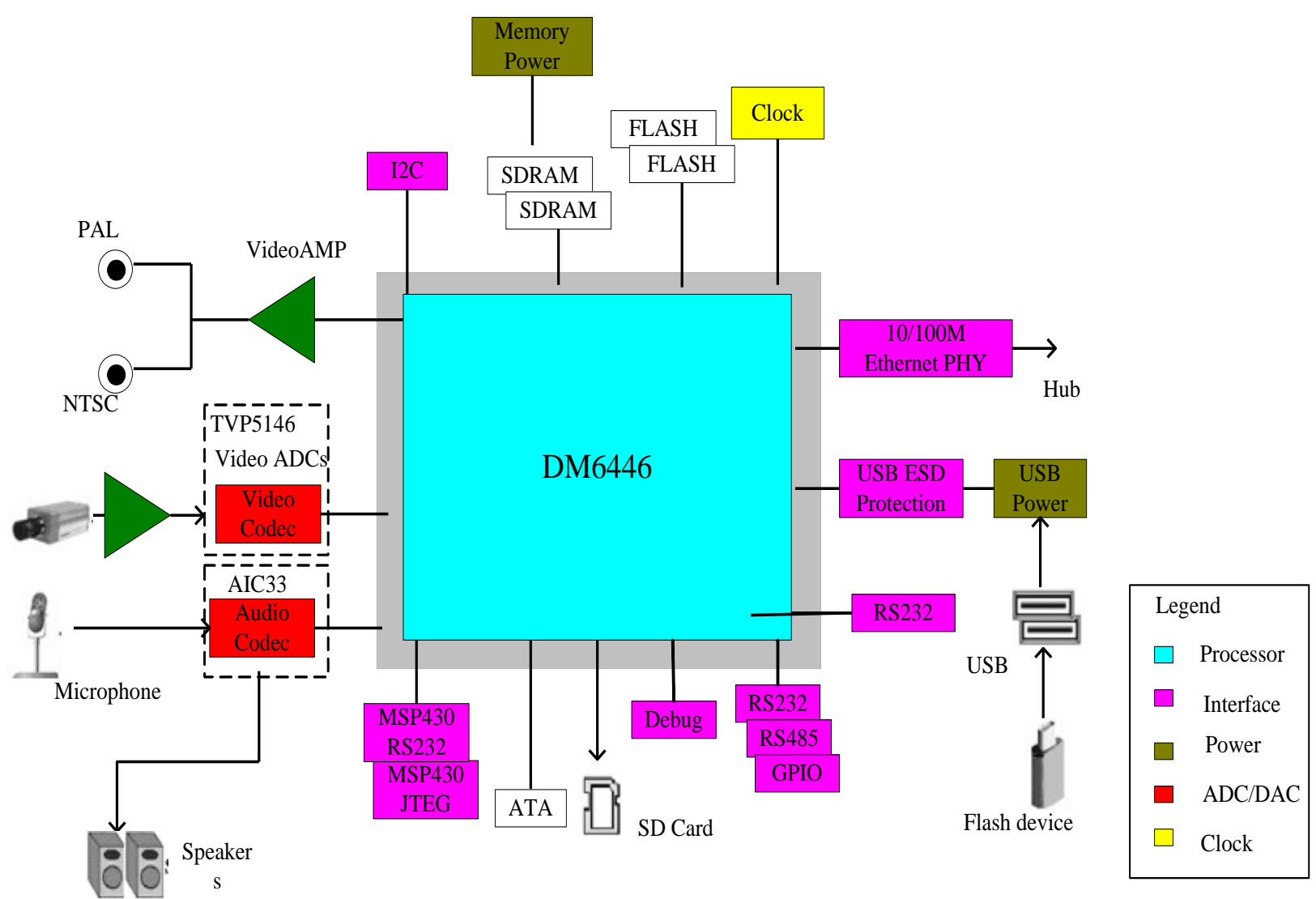

Fig. 7 The Hardware Architecture of DM6446 


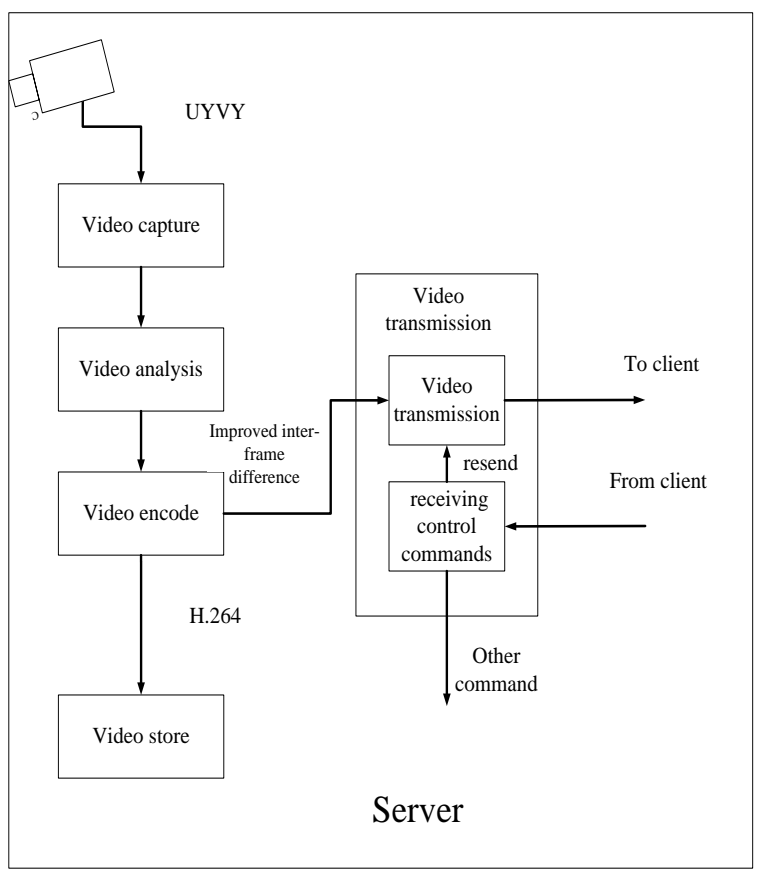

Fig. 8 Video Server Architecture

The video server is designed based on DM6446 software framework. Fig. 9 shows the threads of the application which is designed by Linux multi-thread.

It consists of a main thread (lead to control thread at last), a capture thread, an analysis thread, a video thread and a trans thread. The main thread completes the initialization of the program and provides global parameters to create other threads. Capture thread picks up video in UYVY format by the V4L2 framework. V4L2 is short for Video For Linux 2, which is the driven framework of controlling video equipment on Linux, and it establishes a standard control flow for application program to call [29].Analysis thread includes image process which applies the improved inter-frame difference algorithm for moving tidal detection. Video thread encodes video data for trans thread, which sends the encoded video data to the client over the network. Throughout the process, the data exchanges frequently among the threads. Fig. 10 shows the process of data communication among the threads.

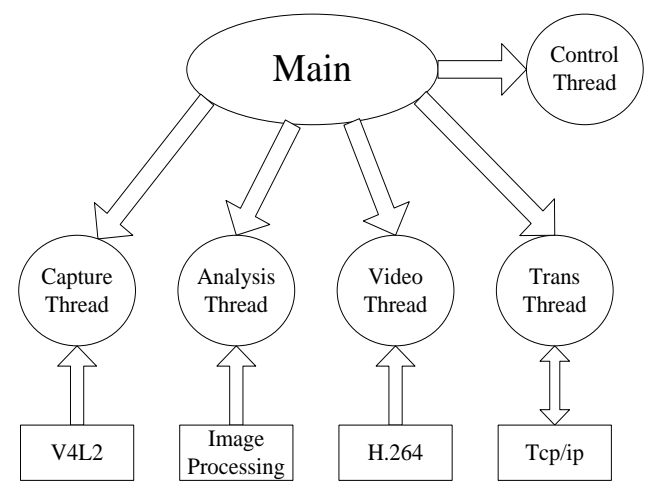

Fig. 9 The Threads of the Program

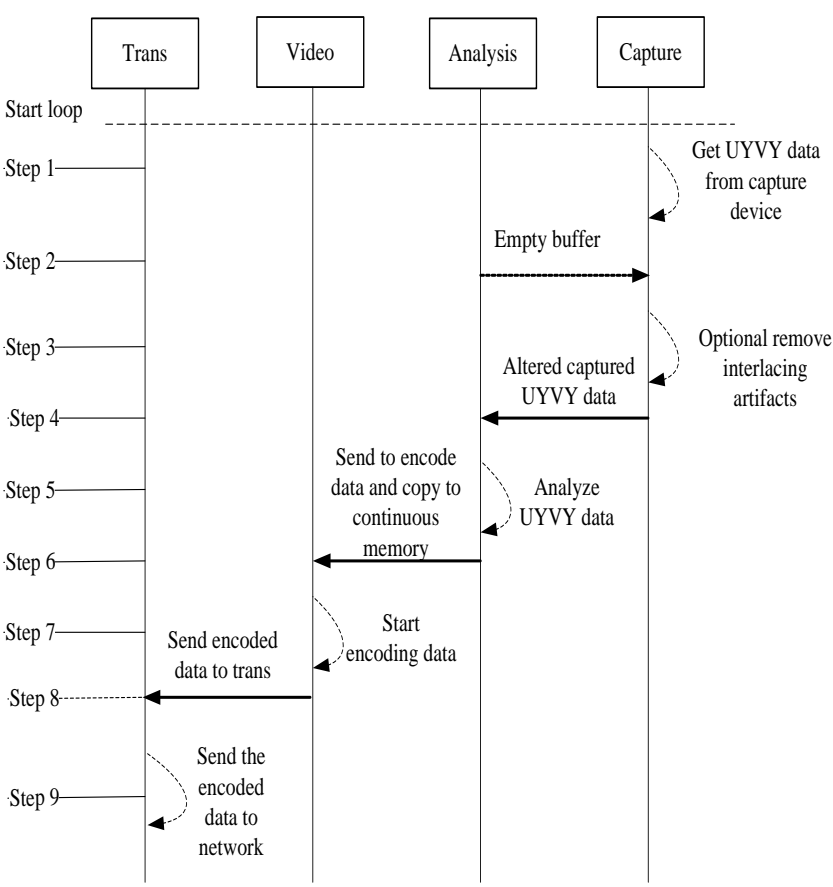

Fig. 10 Thread Data Communication

\section{The implementation of client}

On the client, there also exist many different modules which include the information exchange module for message deliver, the data video receiver module for the receipt of video coding from H.264 compression algorithm, and the transmit Modules for sending control command from PC. The main function of video decode module is to restore the video information after coding. Video display module is developed by DirectShow which is based on Microsoft multimedia development framework. Its main function is to simultaneously display multi-channel and deliver the multivideo to video render for rendering. The client architecture is shown in Fig. 11. And Fig. 12 shows the main process of client program.

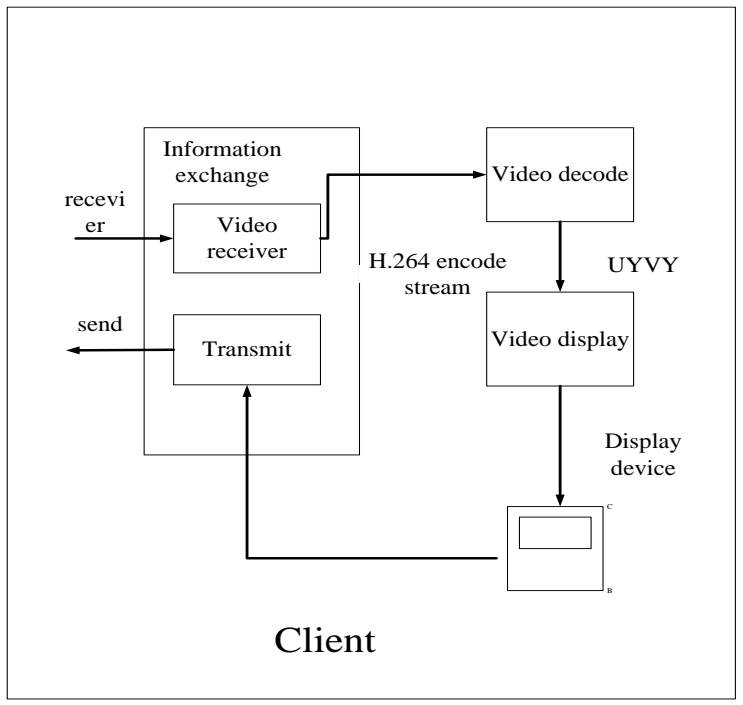

Fig. 11 Client Architecture 


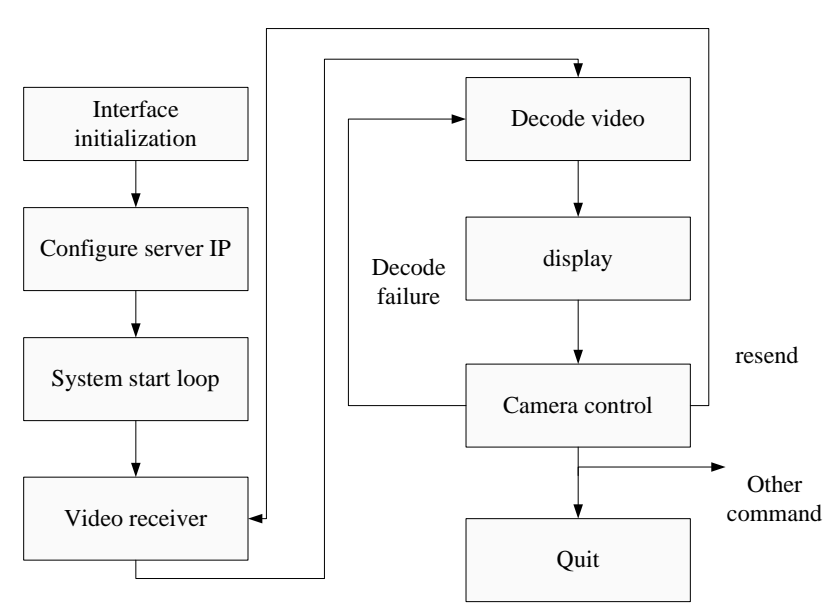

Fig. 12 Client Architecture

Client interface development is completed by MFC which provides a set of controls and flexible messaging and communication mechanisms. It can efficiently enhance the user experience of surveillance system and the humancomputer interaction task can be better performed.

The decoding module of the client should correspond with the code module of the video server. The video server encodes the data by DSP and use H.264 compression algorithm, which means that the decoding module must be able to decode H.264 format. Ffmpeg offers a variety of decoding algorithms including MPEG1, MPEG4 and H.264,and provides a flexible and efficient decoding speed even for upper computer which don' t support hardware decoding .Thus it not only can reduce the delay of the monitoring system, but also benefit the development of the system. Meanwhile, ffmpeg can easily apply to Windows system for MFC developing applications. The process of decoding is shown in Fig. 13. And the architecture of DirectShow is shown in Fig. 14.

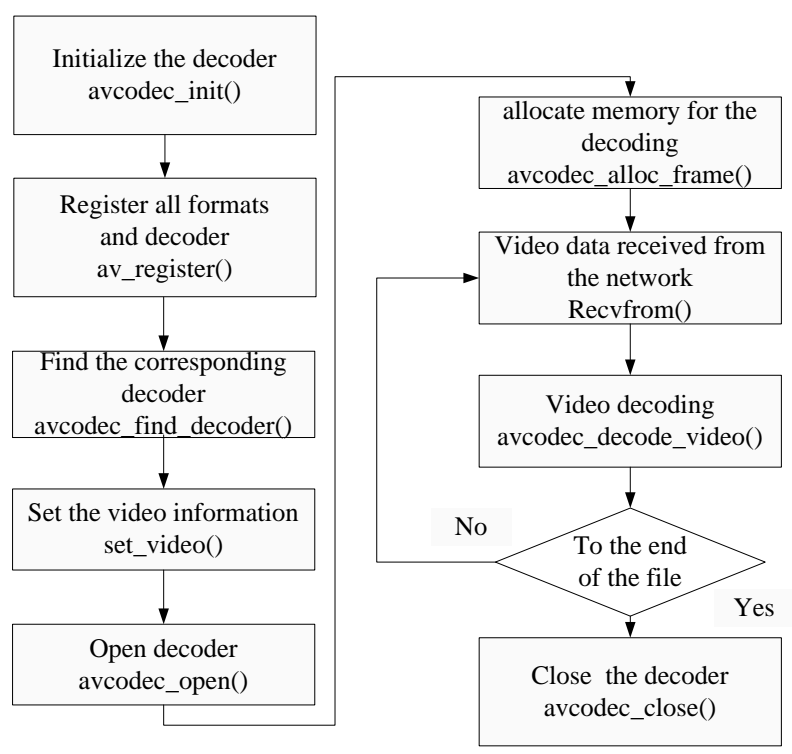

Fig. 13 The Process of ffmpeg

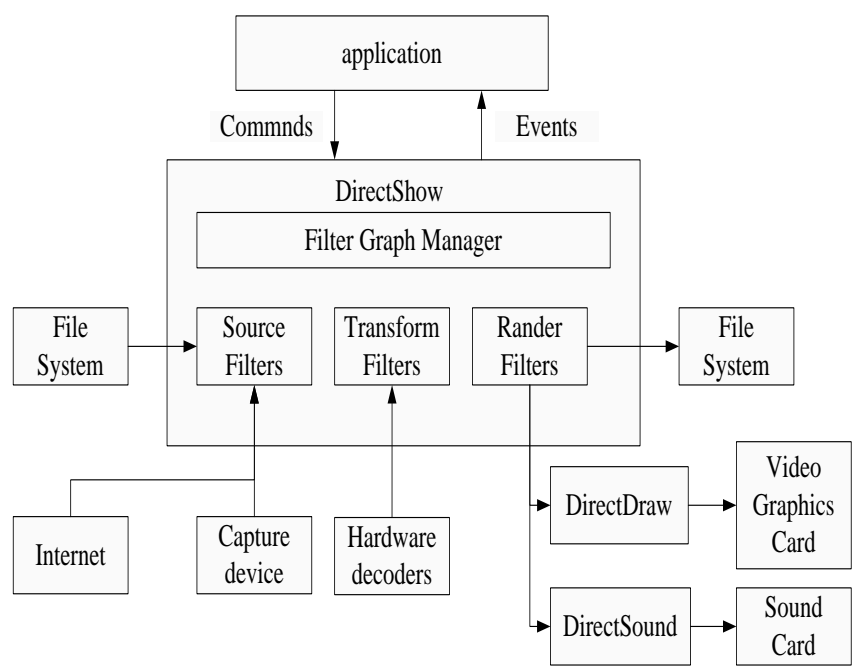

Fig. 14 DirectShow Architecture

Fig. 14 implies how the DirectShow works. The display module functions by SDL which is self-contained in ffmpeg, whose official example is also SDL. SDL displays well in itself and supports message communication mechanism. Meanwhile, it can respond to all kinds of IO- input of keyboard and mouse. However, it only support single picture channel, so it can not meet the multi-channel and multi-screen requirements of video monitoring system. Therefore, the system uses multi-media processing suite named DirectX which is provided by Microsoft especially for Windows. As a part of DirectX, DirectShow is specially used for the making of display frame, and can effectively bind multiple channels with MFC control. To be exactly, it operates fluently in 8,9 , and even 16 picture channels under the support of GPU, and can custom components according to the requirements of a given task. DirectShow realize a seamless connection with MFC and make it easy to maintain and extend the system in the future.

Fig. 15 is the development model of DirectShow in general condition. Among them, the Source Filters receive video from the video server; the Transform Filters is a package of ffmpeg decoder; the Render Filter is used for display. These Filters compose a graph, and if there are multiple server connections, it will build corresponding parallel graphs. To your attention, the Source Filters use "push" mode which will take the initiative to push the encoded data to next Filter after video data is finished receiving.

\section{Experiment Result}

The video server is running on DM6446 while the client uses PC with the hardware configuration of Intel Pentium(R) $1.73 \mathrm{GHz}$, DDR2 1G, Intel GMA900 graphics on Windows XP. The result is as Fig. 16-19 shows.

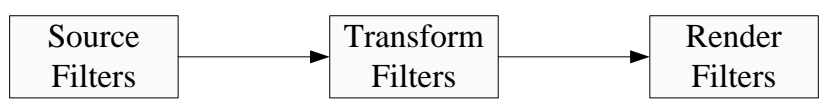

Fig. 15 DirectShow Development Model 


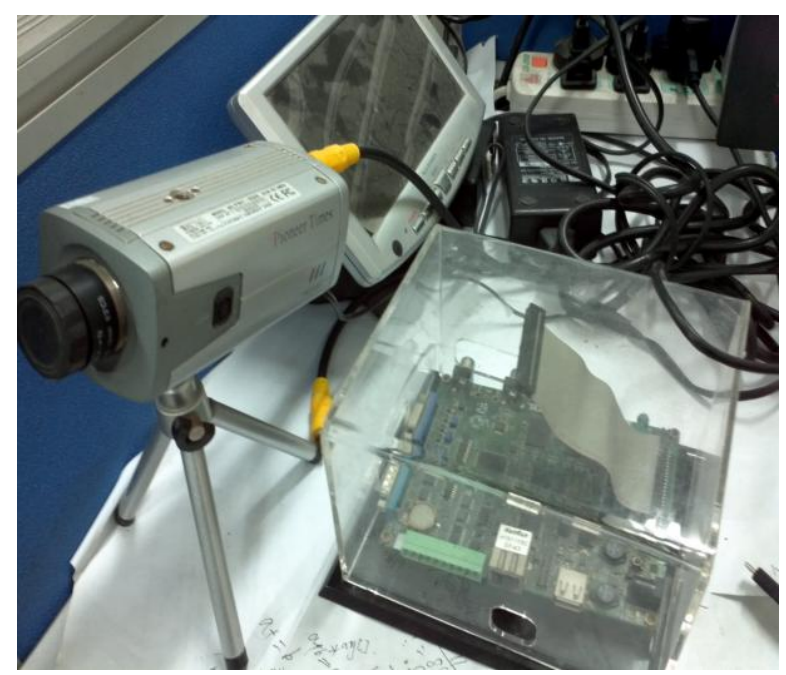

(a) DM6446 DVS

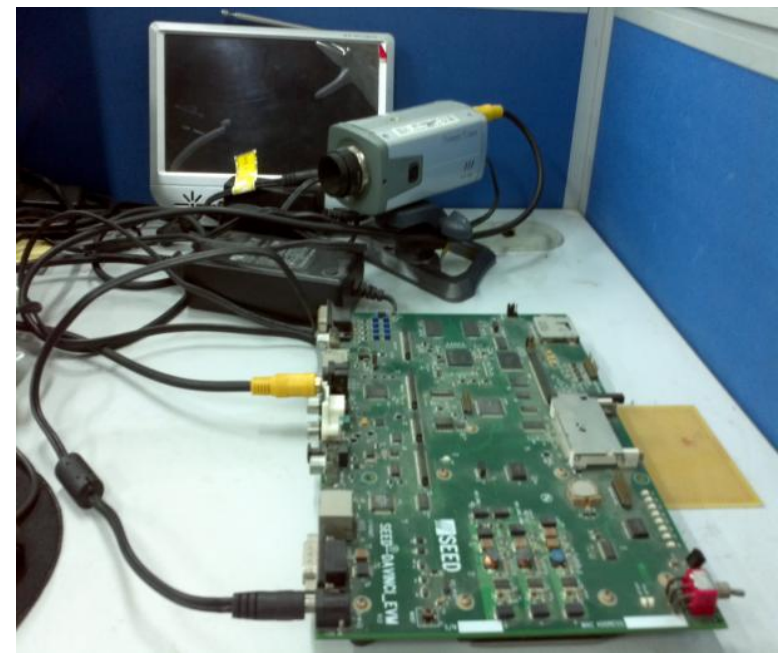

(b) DM6446 EMV

Fig. 16 DM6446 Development Board

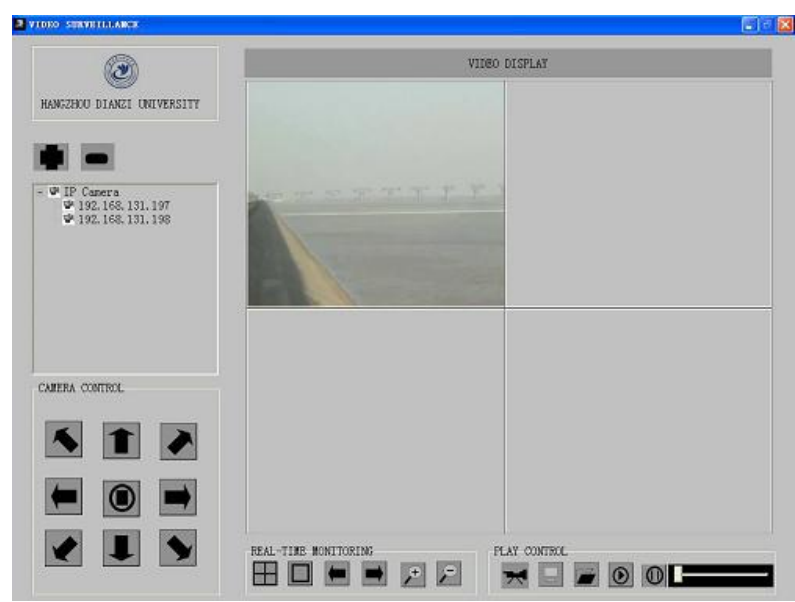

Fig. 17 The Client Interface

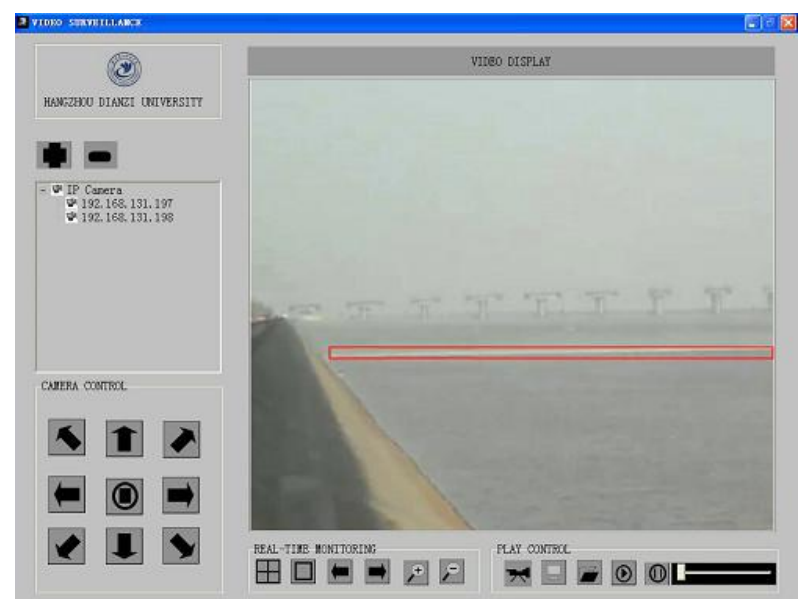

Fig. 18 The Previous Moving Tidal Detection Result

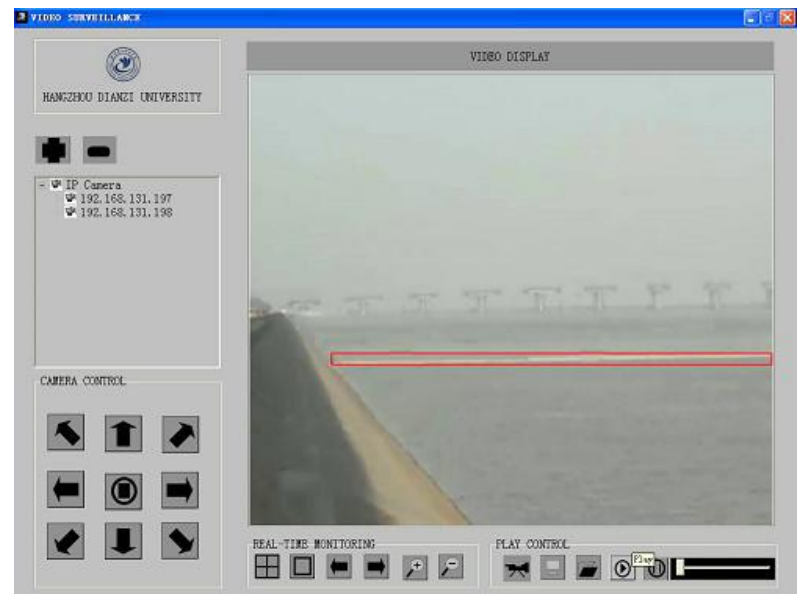

Fig. 19 The Moving Tidal Detection Result

System test shows that in area within the monitoring range of camera, the moving objects can be detected with a delay of 300-500 milliseconds. The network can provide enough bandwidth to perform multi-channel video transmission task due to the high compression rate of H.264. The fact that the system can also run the client even in poorconfiguration $\mathrm{PC}$ proves that the intelligent video surveillance system we present will take up no more PC hardware resources than the traditional video surveillance system. Thus, it gets rid of the restrictions of the requirements of the hardware equipment performance, saves the cost for manufacturers and customers, improves the production efficiency and practical benefit and provides conditions for its further popularization and application.

\section{Conclusions}

An intelligent video surveillance system based on DM6446 was presented in this paper which is applied to realize automatic tidal bore video recognition for the first time. Combined with digital image process technology, this prototype implementation applies an improved inter-frame difference algorithm to detect tidal bore, which is proved to be better than the other two previous algorithms. As the 
experiment shows, each module of the system works normally and operates stably, leading to effective detection of the front end of the tidal bore in Qiantang River. Moreover, it can perform intelligent monitoring with low delay, achieving the goal of real-time monitoring and supporting the supervision work of security personnel. All of those suggest a good commercial value.

\section{Acknowledgment}

This work was supported by Project supported by the National Natural Science Foundation of China (No. 61374005), Hangzhou Hydrology and Water Resources Monitoring Station and Hangzhou Rural Water Management Main Station.

\section{References}

[1] Lin B Y, "Characters of Qiantang Bores (in Chinese)," Beijing: Ocean Press, 2008.

[2] WOLANSKI E., WILLIAMS D. and SPAGNOL S. et al, "Undular tidal bore dynamics in the Daly Estuary, Northern Australia. Estuarine," Coastal and Shelf Science, vol. 60, no. 4, pp. 629-636, 2004.

[3] Huang, J., Pan, C.,H., Kuang, C.,P., Zeng,J., Chen,G., "Experimental hydrodynamic study of the Qiantang River tidal bore," Journal of Hydrodynamics, vol. 25, no. 3, pp. 481-490, 2013.

[4] Pan,C.,H., Lin, B.,Y., and MAO X., "Case study: Numerical modeling of the tidal bore on the Qiantang River, China," Journal of Hydraulic Engineering, ASCE, vol. 133, no. 2, pp. 130-138, 2007.

[5] Wang, R., R.,Wang, J.,Z.,Xue, A.,K., "A tidal bore detecting method based on audio frequency recognition," STATE INTELLECTUAL PROPERTY OFFICE OF THE P.R.C, ZL200810121881.7, 2010.

[6] Wang, R., R.,Wang, J.,Z.,Xue, A.,K., "A single-shaft tidal current velocity measuring device," STATE INTELLECTUAL PROPERTY OFFICE OF THE P.R.C, ZL201020108649.2, 2010.

[7] Wang, R., R.,Wang, J.,Z.,Xue, A.,K., “A double-shaft tidal current velocity measuring device," STATE INTELLECTUAL PROPERTY OFFICE OF THE P.R.C, ZL2010201086789.9, 2010.

[8] Xue, A.,K., Wang, R., R.,Wang, J.,Z., "A method of predicting tidal water level and time based on neural network," STATE INTELLECTUAL PROPERTY OFFICE OF THE P.R.C, ZL200910101081.3, 2010.

[9] Wang, R., R.,Wang, J.,Z.,Xue, A.,K., "A method of real-time tidal bore prediction by dividing the river into many segments," STATE INTELLECTUAL PROPERTY OFFICE OF THE P.R.C, ZL200810164101.7, 2010.

[10] E.Malamas, E.Petrakis, M.Zervakis, L.Petit, "J.D.Legat. A Survey on Industrial Vision Systems, Applications and tools," Image and Vision Computing, vol. 21, pp. 171-188, 2003.

[11] Jiang Feng-jiao, Zhao Xiao-feng, Zhao Shu-ping, Li-li Dong, "Research on Infrared target tracking and detection based on Least
Squared method," Electrical and Control Engineering International Conference, pp. 25-27, June 2010.

[12] Anna Petrovskaya, Sebastian Thrun, "Model based vehicle detection and tracking for autonomous urban driving," Autonomous Robots, vol. 26, pp. 123-139, 2009.

[13] Remagnino P, Tan T, Baker K, "Multi-agent visual surveillance of dynamic scenes," Image and Vision Computing, vol. 16, no. 8, pp. 529$532,1998$.

[14] Jun-Wei Hsieh, Yung-Tai Hsu, Hong-Yuan Mark Liao, and ChihChiang Chen, "Video-Based Human Movement Analysis and Its Application to Surveillance Systems," IEEE TRANSACTIONS ON MULTIMEDIA, vol. 10, no. 3, pp. 372-384, 2008

[15] ITU-T Recommendation H.261, "Video Codec for Audio visual Services at p*64kbit/s," 1990.

[16] ISO/IEC International Standard 11172, "Coding of Moving Pictures and Associated Audio for Digital Storage Media at up to about 1.5Mbit/s -part2: video," 1993.

[17] ISO/IEC International Standard 13818, "Generic Coding of Moving Pictures and Associated Audio," 1995.

[18] ITU-T Recommendation H.263, "Video Coding for Low Bitrate Communication Version 1," 1995.

[19] ITU-T Standardization Sector of ITU, "Video coding for low bit rate communication, Draft ITU-T Recommendation H.263 2,” ITU-T, 1998.

[20] ISO/IEC International standard 14496, "Coding of Moving Pictures and Audio," 1999.

[21] ITU-T ISO/IEC 14496-10 AVC, "Draft ITU-T Recommendation and Final Draft Internation Standard of Joint Video Specification," 2003.

[22] Thomas Wiegand, Michael Lightstone et al, "Rate-Distortion Optimizated Mode Selection for very low Bit Rate Coding and the Emerging H.263 Standard," IEEE Trans. Circuits and Systems for Video Technology, vol. 6, pp. 182-190, April 1996.

[23] Yu-Han Chen, Tung-Chien Chen, Chuan-Yung Tsai, Sung-Fang Tsai, Liang-Gee Chen, "Algorithm and Architecture Design of PowerOriented H.264/AVC Baseline Profile Encoder for Portable Devices," Circuits and Systems for Video Technology, vol. 19, no. 8, pp. 11181128,2009

[24] Wiegand T, Sullivan G.J, Bjontegaard G, Luthra A, "Overview of the H.264/AVC video coding standard," Circuits and Systems for Video Technology, vol. 13, no. 7, pp. 560-576, 2003.

[25] Kim Y.-H, Yoo J.-W, Lee S.-W, Shin J, Paik J, Jung H.-K, “Adaptive mode decision for H.264 encoder," Electronics Letters, vol. 40, no. 19, pp. 1172-1173, 2004.

[26] Mahadevan V, Vasconcelos N, "Background subtraction in highly dynamic scenes," Computer Vision and Pattern Recognition IEEE Conference, 2010

[27] Zha Yufei, Bi Duyan, "Adaptive learning algorithm based on mixture Gaussian background," Journal of System Engineering and Electronics, vol. 18, pp. 369-376, 2007.

[28] Rui Liang, Lei Yan, Pengqi Gao, Xu Qian, Zhongjian Zhang, Huabo Sun, "Aviation video moving-target detection with inter-frame difference," Image and Signal Processing International Congress, pp. 1494-1497, 2010

[29] Michael H Schimek, Bill Dirks, Hans Verkuli, "Video for Linux two API specification," http://v412spec.bytesex.org/v412spec/v412. pdf, 2008. 